\title{
5 Characteristics of He ion implanted layers on single-crystal
}

diamond

Audrey Valentin ${ }^{1}$, Mary De Feudis ${ }^{1,2}$, Ovidiu Brinza ${ }^{1}$, André Tardieu ${ }^{1}$, Riadh Issaoui ${ }^{1}$, Ludovic William ${ }^{1}$, Jocelyn Achard ${ }^{1}$ LSPM-CNRS, Laboratoire des Sciences des Procédés et des Matériaux, Université Paris 13, Sorbonne Paris Cité, avenue Jean-Baptiste Clément, 93430 Villetaneuse, France ${ }^{2}$ Dep. Mathematics and Physics, University of Salento, 73100 Lecce, Italy

\section{Background and aim of the study}

Context:

- Heavy ion implantation used for

contact fabrication [1]

- He ion implantation for $\mathrm{N}-\mathrm{V}$

centers [2]

He ion implantation for the enhancement of ohmic contacts 1st result [3]: ohmic contact fabricated on He ion-implanted boron-doped samples

$\rightarrow$ ohmicity improved

$\rightarrow$ contact resistance decreased

$\rightarrow$ ohmic contacts obtained even at a doping level of $2.10^{17} \mathrm{~cm}^{-3}$

Aim of the study: characteristics of He ion-implanted single-crystal diamond.

\section{Ion implantation}

Experimental: He ion implantation at various fluences (from $4 \times 10^{13}$ to $5.5 \times 10^{15}$ ions $/ \mathrm{cm}^{2}$ ) with a home-made low-cost ion implanter.

One of the sample corners is masked during the implantation process.

Several annealing steps are performed after the implantation process.

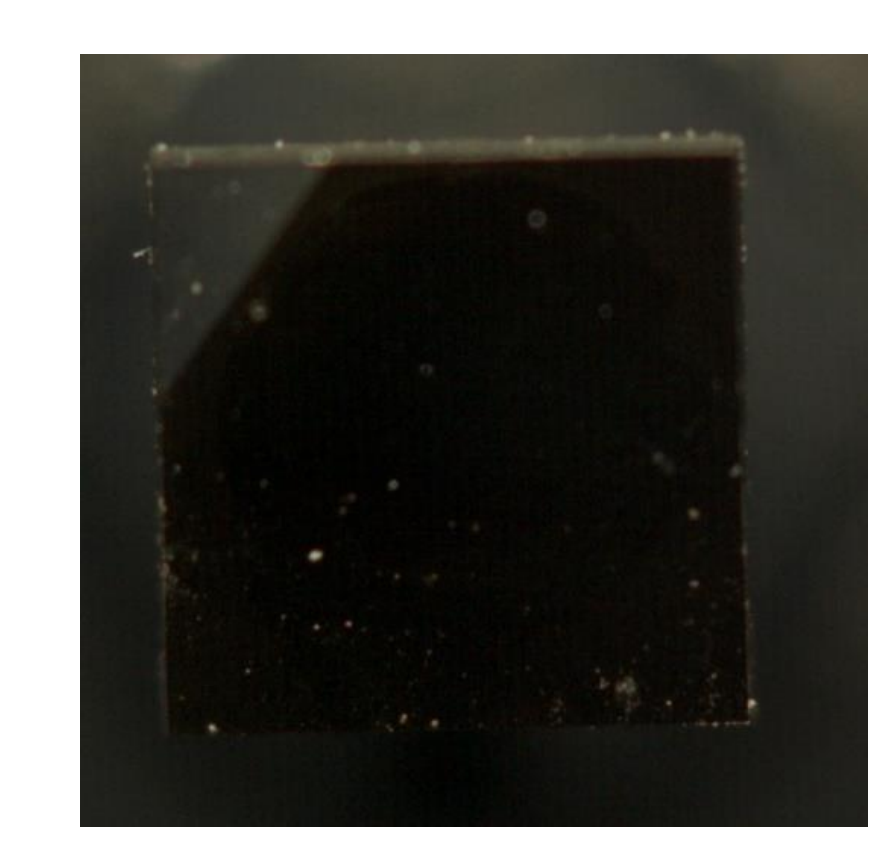

Diamond View images of an implanted sample.

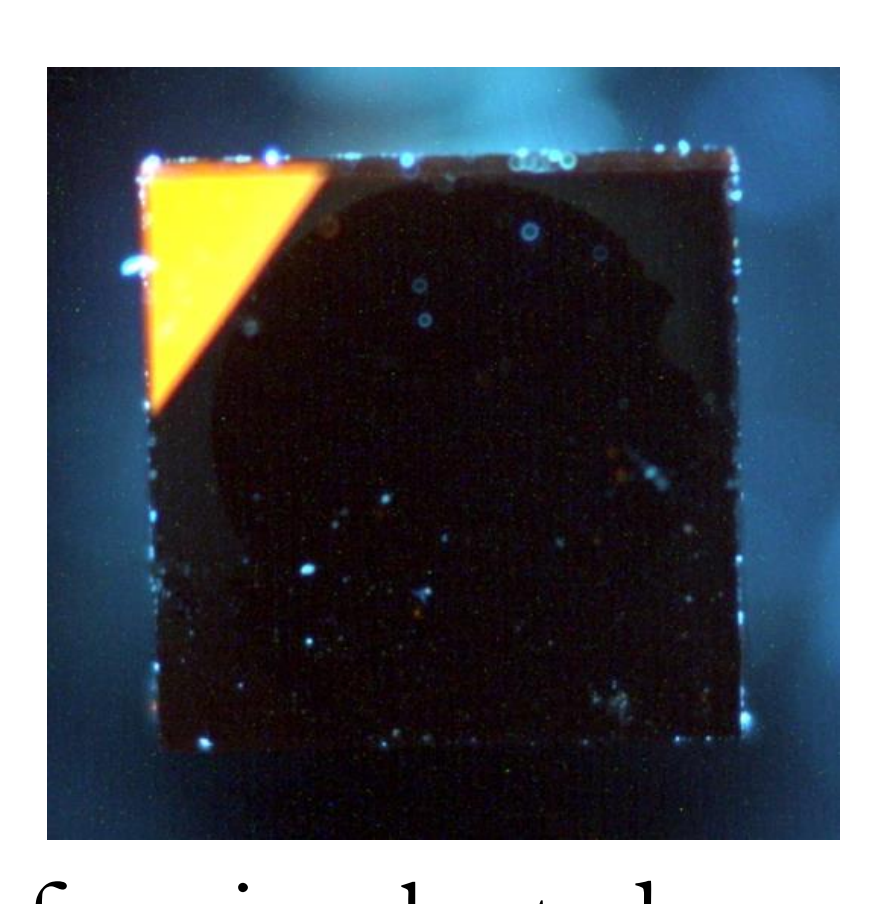

SRIM simulation of implantation:

- 15 vacancies/ion

- $50 \mathrm{~nm}$ penetration depth

$\rightarrow$ The density of vacancies is above

the amorphisation threshold for considered fluences.

\section{Density profiling}

Experimental: Surface profile measured by confocal microscopy (Keyence VK 9700)

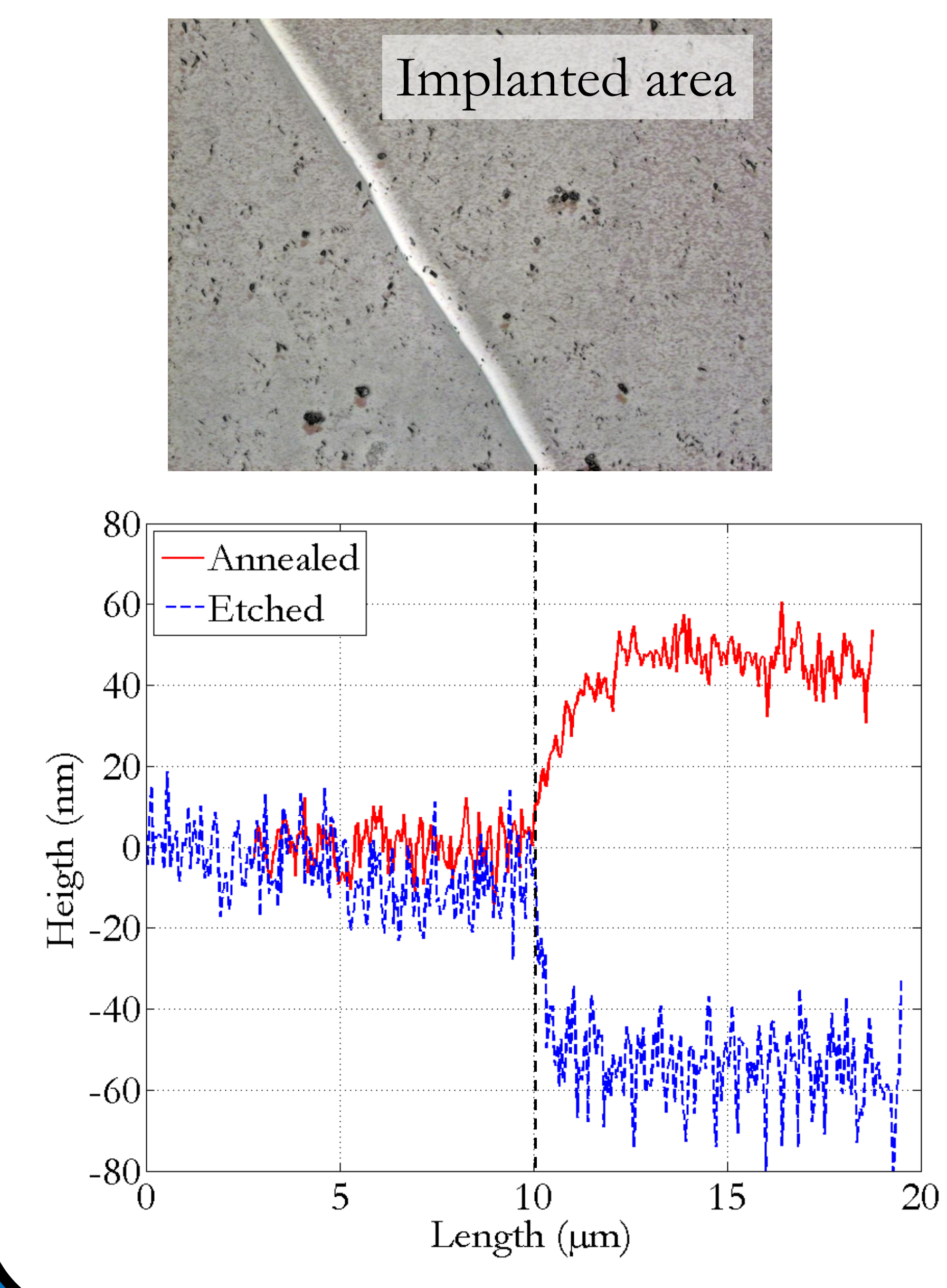

- Before annealing, the effect on density is not observable

- After annealing, a step $\sim+50 \mathrm{~nm}$ appears.

- After etching, a step $\sim-60 \mathrm{~nm}$ is revealed.

$\rightarrow$ The layer has the same density as graphite.

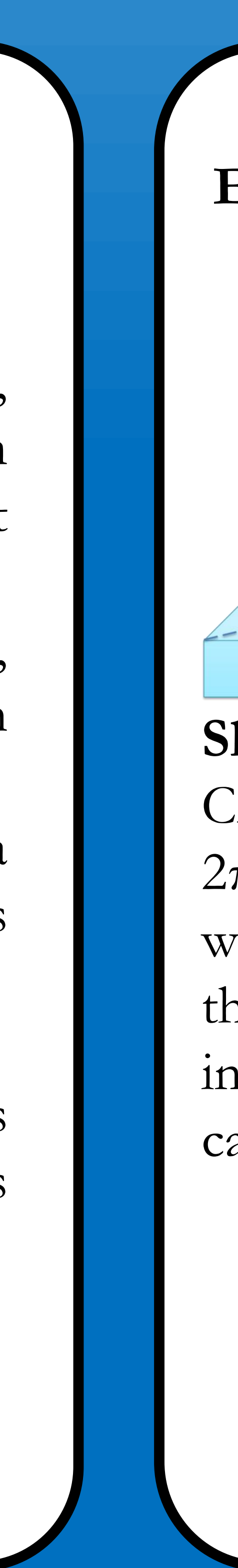

\section{Resistivity measurement}

Experimental: 4-point probe resistivity measurement using a Keithley 4200 semiconductor characterization system.

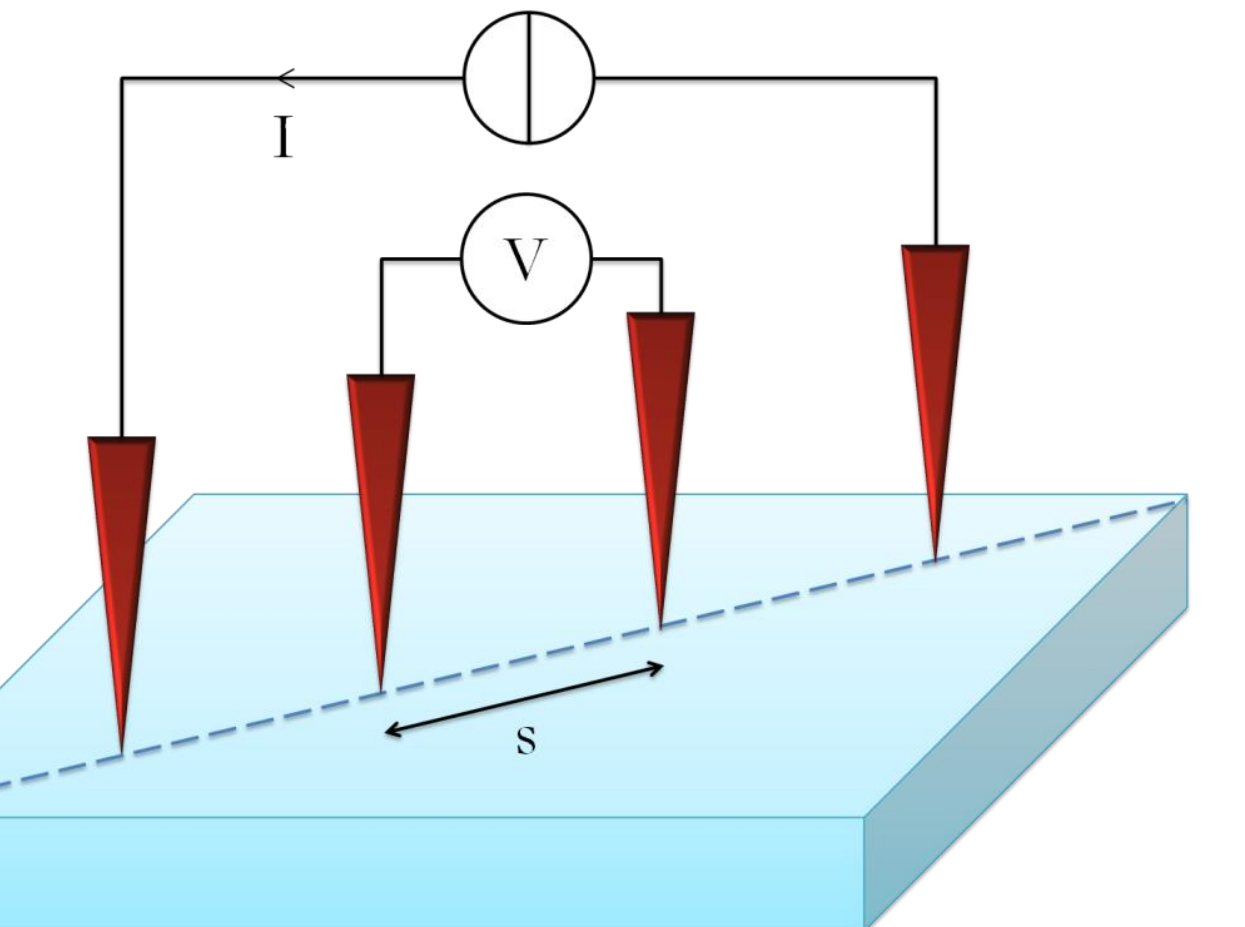

Sheet resistance evaluation:

Classical formula $\varrho=$

$2 \pi \mathrm{Fs} . \mathrm{V} / \mathrm{I}$

where $\mathrm{F}$ is calculated using

the procedure detailed in [4]

in our specific geometric case.
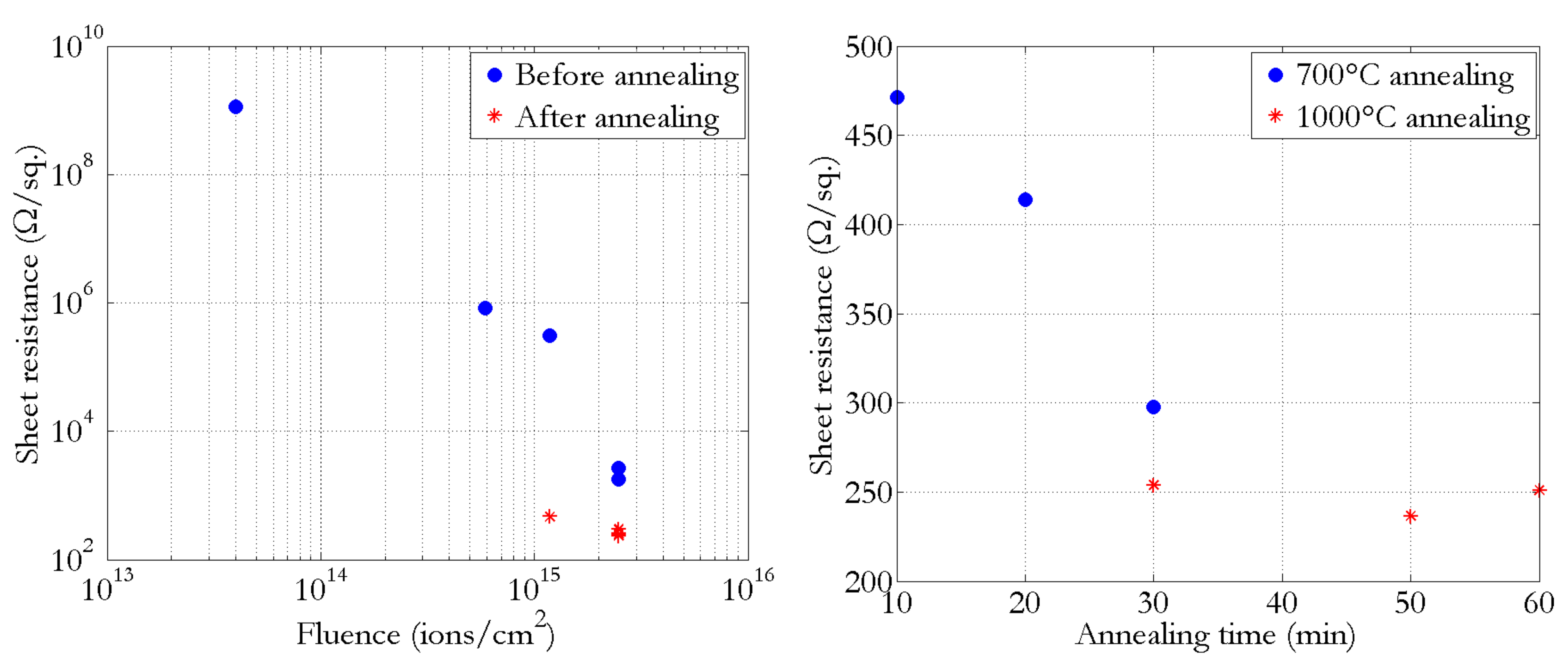

$\rightarrow$ Before implantation, all the samples are insulating.

$\rightarrow$ Before annealing : resistivity decreases with increasing the resistivity.

ion fluence.

$\rightarrow$ Annealing decrease the resistivity

$\rightarrow$ The final sheet resistance is close to the graphite sheet resistance.

\section{Raman spectra}

Experimental: Spectra acquired using a Horiba HR800 spectrometer @RT - 473 nm.

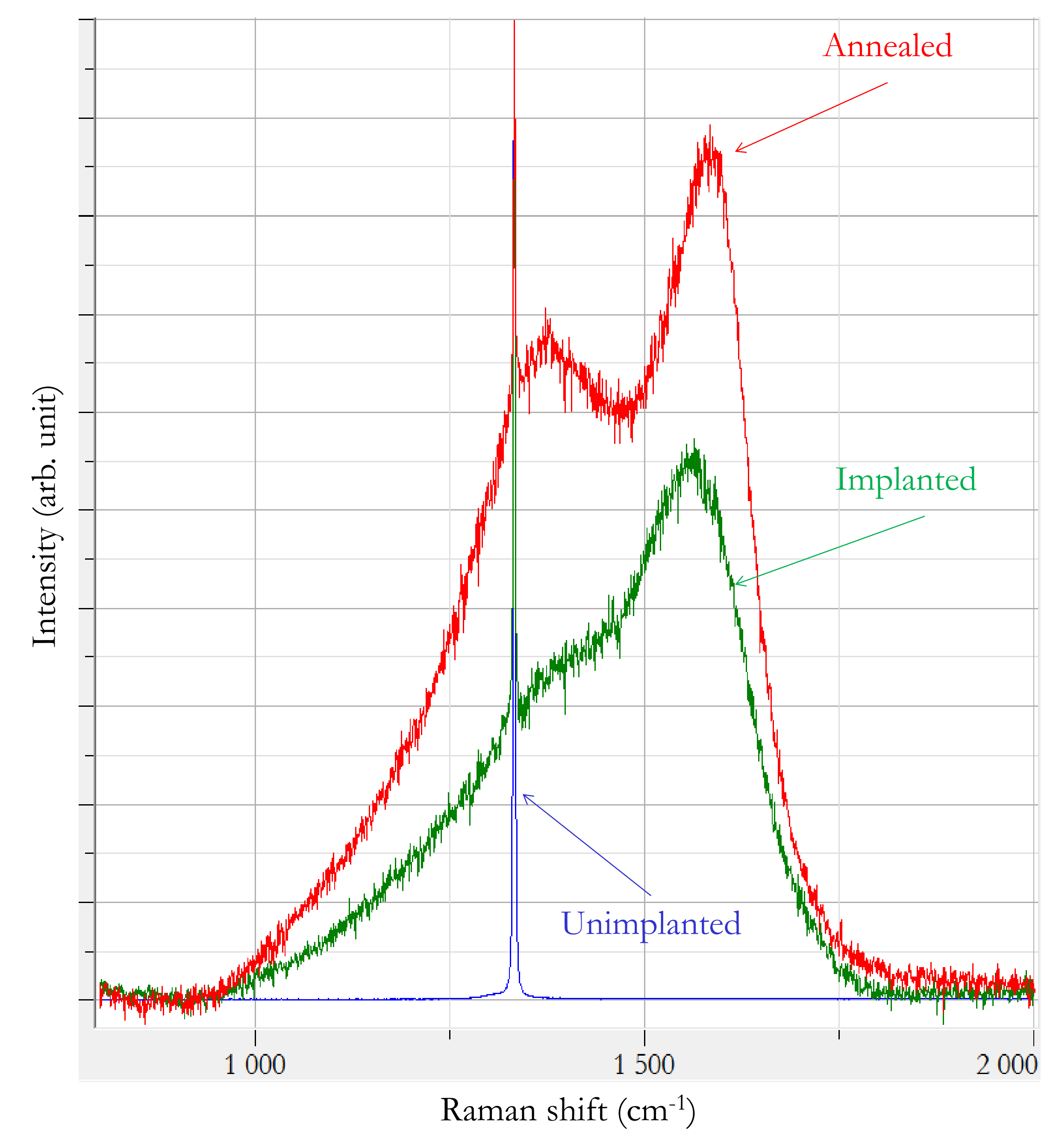

$\rightarrow$ Implantation induces the appearance of the G-band and the D-band: coexistence of disordered graphite and diamond.

$\rightarrow$ Annealing shifts the G-band et D-band and decreases their width.

$\rightarrow$ The G-band position shifts from 1555-1570 $\mathrm{cm}^{-1}$ (amorphous carbon) to $1585-1595 \mathrm{~cm}^{-1}$ (nanocrystalline graphite).
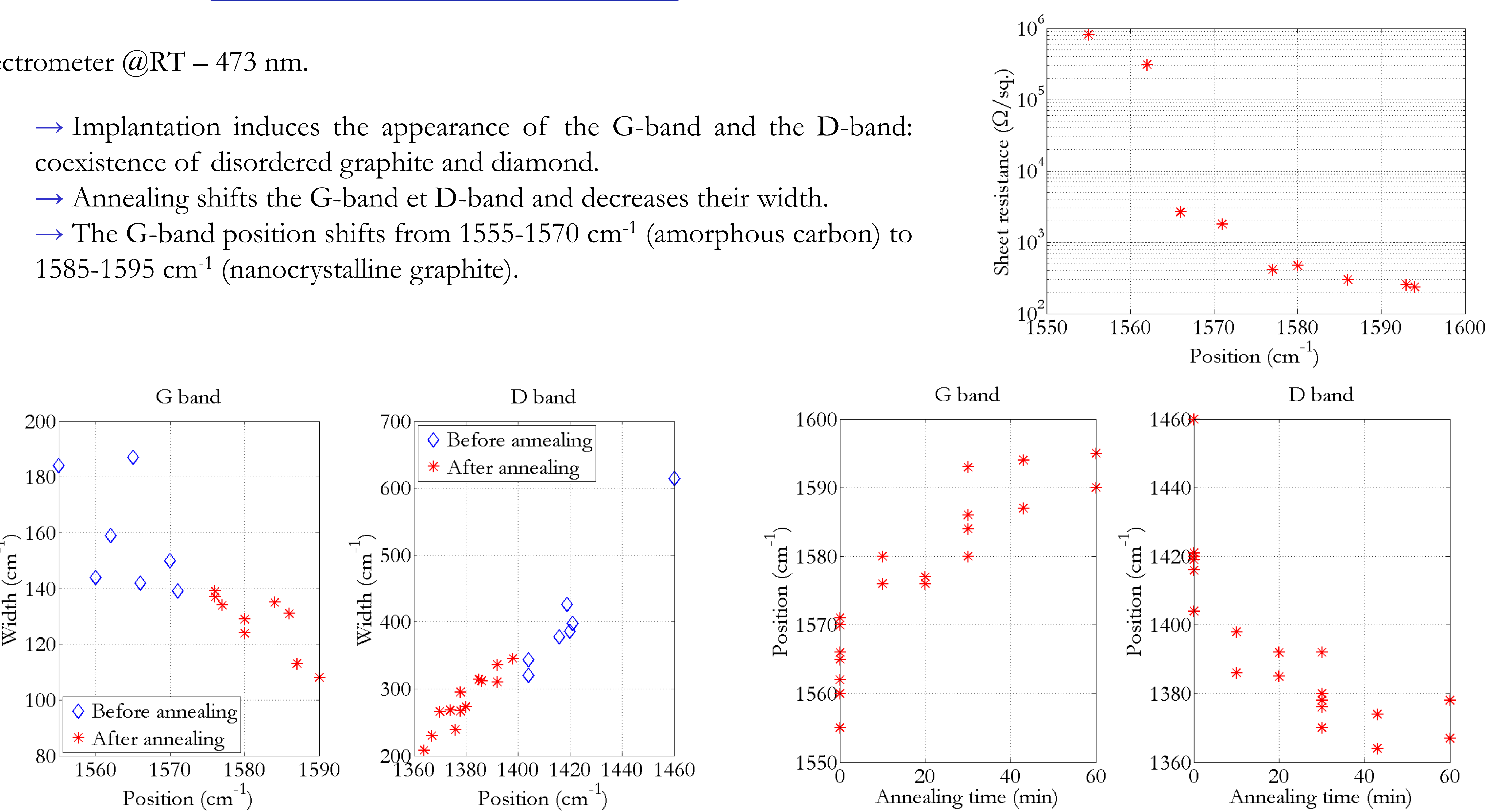

\section{References}

